\title{
Quantum Statistical Derivation of a Ginzburg-Landau Equation
}

\author{
Shigeji Fujita1, Akira Suzuki² \\ ${ }^{1}$ Department of Physics, University at Buffalo, State University of New York, Buffalo, USA \\ ${ }^{2}$ Department of Physics, Faculty of Science, Tokyo University of Science, Tokyo, Japan \\ Email: asuzuki@rs.kagu.tus.ac.jp
}

Received 4 August 2014; revised 1 September 2014; accepted 26 September 2014

Copyright (C) 2014 by authors and Scientific Research Publishing Inc.

This work is licensed under the Creative Commons Attribution International License (CC BY).

http://creativecommons.org/licenses/by/4.0/

(c) (i) Open Access

\section{Abstract}

The pairon field operator $\Psi(r, t)$ evolves, following Heisenberg's equation of motion. If the Hamiltonian $H$ contains a condensation energy $\alpha_{0}(<0)$ and a repulsive point-like interparticle interaction $\beta_{0} \delta\left(r_{1}-r_{2}\right), \beta_{0}(>0)$, the evolution equation for $\Psi$ is non-linear, from which we derive the Ginzburg-Landau (GL) equation: $\alpha_{0} \Psi_{\sigma}(r)+\beta_{0}\left|\Psi_{\sigma}(r)\right|^{2} \Psi_{\sigma}(r)=0$ for the GL wave function $\Psi_{\sigma}(r) \equiv\langle r|u| \sigma\rangle, n \equiv u u^{\dagger}$ where $\sigma$ denotes the state of the condensed Cooper pairs (pairons), and $n$ the pairon density operator ( $u$ and $u^{\dagger}$ are kind of square root density operators). The GL equation with $\alpha_{0}=-\varepsilon_{g}(T)$ holds for all temperatures $(T)$ below the critical temperature $T_{c}$, where $\varepsilon_{g}(T)$ is the $T$-dependent pairon energy gap. Its solution yields the condensed pairon density $n_{0}(T)=\left|\Psi_{\sigma}(r)\right|^{2}=\beta_{0}^{-1} \varepsilon_{g}(T)$. The $T$-dependence of the expansion parameters near $T_{c}$ obtained by GL: $\alpha_{0}=-c\left(T_{c}-T\right), \quad \beta_{0}=$ constant is confirmed.

\section{Keywords}

Ginzburg-Landau Equation, Complex-Order Parameter, Coherent Length, Cooper Pair (Pairon), Pairon Density Operator, T-Dependent Pairon Energy Gap

\section{Introduction}

In 1950 Ginzburg and Landau (GL) [1] proposed a revolutionary idea that below the critical temperature $T_{c}$ a 
superconductor has a complex order parameter (also known as a GL wave function) $\Psi$ just as a ferromagnet possesses a real order parameter (spontaneous magnetization). Based on Landau's theory of second-order phase transition [2], GL expanded the free energy density $f(\boldsymbol{r})$ of a superconductor in powers of small $|\Psi(\boldsymbol{r})|$ and $|\nabla \Psi(\boldsymbol{r})|:$

$$
f(\boldsymbol{r})=f_{0}+\alpha_{0}|\Psi(\boldsymbol{r})|^{2}+\frac{1}{2} \beta_{0}|\Psi(\boldsymbol{r})|^{4}+\frac{\hbar^{2}}{2 m_{0}}|\nabla \Psi(\boldsymbol{r})|^{2},
$$

where $f_{0}, \alpha_{0}$ and $\beta_{0}$ are constants, and $m_{0}$ is the superelectron mass. To include the effect of a magnetic field $\boldsymbol{B}$, they used a quantum replacement:

$$
\nabla \rightarrow \nabla-(i q / \hbar) A, \quad q=\text { charge },
$$

where $\boldsymbol{A}$ is a vector potential generating $\boldsymbol{B}=\nabla \times \boldsymbol{A}$, and added a magnetic energy term $\boldsymbol{B}^{2} / 2 \mu_{0}$. The integral of the free energy density $f(\boldsymbol{r})$ over the sample volume $\Omega$ gives the Helmholtz free energy $F$. After minimizing $F$ with variation in $\Psi^{*}$ and $\boldsymbol{A}_{j}$, GL obtained two equations:

$$
\begin{gathered}
\frac{1}{2 m_{0}}(-i \hbar \nabla-q \boldsymbol{A})^{2} \Psi(\boldsymbol{r})+\alpha_{0} \Psi(\boldsymbol{r})+\beta_{0}|\Psi(\boldsymbol{r})|^{2} \Psi(\boldsymbol{r})=0, \\
\boldsymbol{j}=-\frac{i q \hbar}{2 m_{0}}\left(\Psi^{*} \nabla \Psi-\Psi \nabla \Psi^{*}\right)-\frac{q^{2}}{2 m_{0}} \Psi^{*} \Psi \boldsymbol{A},
\end{gathered}
$$

with the density condition:

$$
\Psi^{*}(\boldsymbol{r}) \Psi(\boldsymbol{r})=n_{\mathrm{s}}(\boldsymbol{r})=\text { superelecton density },
$$

where $\boldsymbol{j}$ is the current density.

The superelectron model has difficulties. Since electrons are fermions, no two electrons can occupy the same particle state by Pauli's exclusion principle. Cooper [3] introduced Cooper pairs in 1956. Bardeen, Cooper and Schrieffer (BCS) published a classic paper [4] on the superconductivity in 1957 based on the phonon exchange attraction between two electrons. The center-of-mass (CM) of Cooper pairs (pairons) move as bosons, which is shown in Section 2. We view that the Bose-condensed pairons can generate the superconducting state. We modify the density condition (5) to

$$
\left|\Psi_{0}(\boldsymbol{r})\right|^{2}=\text { condensed pairon density at the state } \sigma \text {. }
$$

Equation (3) is the celebrated Ginzburg-Landau equation, which is quantum mechanical and nonlinear. Since the smallness of $|\Psi|^{2}$ is assumed, the GL equation was thought to hold only near $T_{c}, T_{c}-T \ll T_{c}$. Below $T_{c}$ there is a supercondensate whose motion generates a supercurrent and whose presence generates gaps in the elementary excitation energy spectra. The GL wave function $\Psi$ represents the quantum state of this supercondensate.

In the present work, we derive the GL wave equation microscopically and show that the GL equation is valid for all temperature below $T_{c}$.

\section{Moving Cooper Pairs (Pairons)}

Fujita, Ito and Godoy in their book [5] discussed the moving pairons. We briefly summarize their theory and main results here. The energy $w_{q}$ of a moving pairon can be obtained from

$$
w_{q} a(\boldsymbol{k}, \boldsymbol{q})=\{\varepsilon(|\boldsymbol{k}+\boldsymbol{q} / 2|)+\varepsilon(|-\boldsymbol{k}+\boldsymbol{q} / 2|)\} a(\boldsymbol{k}, \boldsymbol{q})-\frac{v_{0}}{(2 \pi \hbar)^{3}} \int \mathrm{d}^{3} k^{\prime} a\left(\boldsymbol{k}^{\prime}, \boldsymbol{q}\right),
$$

which is Cooper's equation, Equation (1) of his 1956 Physical Review [3]. The prime on the $\boldsymbol{k}^{\text {'-integral means }}$ the restriction on the integration domain arising from the phonon-exchange attraction, see below. We note that the net momentum $\boldsymbol{q}$ is a constant of motion, which arises from the fact that the phonon exchange is an internal process, and hence cannot change the net momentum $\boldsymbol{q}$. The pair wave functions $a(\boldsymbol{k}, \boldsymbol{q})$ are coupled with respect to the other variable $\boldsymbol{k}$, meaning that the exact (or energy-eigenstate) pairon wavefunctions are superposi- 
tions of the pair wavefunctions $a(\boldsymbol{k}, \boldsymbol{q})$.

Equation (7) can be solved as follows. We assume that the energy $w_{q}$ is negative:

$$
w_{q}<0 \text {. }
$$

Then, $\varepsilon(|\boldsymbol{k}+\boldsymbol{q} / 2|)+\varepsilon(|-\boldsymbol{k}+\boldsymbol{q} / 2|)-w_{q}>0$. Rearranging the terms in Equation (7) and dividing it by $\varepsilon(|\boldsymbol{k}+\boldsymbol{q} / 2|)+\varepsilon(|-\boldsymbol{k}+\boldsymbol{q} / 2|)-w_{q}$, we obtain

$$
a(\boldsymbol{k}, \boldsymbol{q})=\left\{\varepsilon(|\boldsymbol{k}+\boldsymbol{q} / 2|)+\varepsilon(|-\boldsymbol{k}+\boldsymbol{q} / 2|)-w_{q}\right\}^{-1} C(\boldsymbol{q}) .
$$

Here

$$
C(\boldsymbol{q}) \equiv \frac{v_{0}}{(2 \pi \hbar)^{3}} \int \mathrm{d}^{3} k^{\prime} a\left(\boldsymbol{k}^{\prime}, \boldsymbol{q}\right)
$$

is $\boldsymbol{k}$-independent. Introducing Equation (9) in Equation (7), and dropping the common factor $C(\boldsymbol{q})$, we obtain

$$
1=\frac{v_{0}}{(2 \pi \hbar)^{3}} \int \mathrm{d}^{3} k\left\{\varepsilon(|\boldsymbol{k}+\boldsymbol{q} / 2|)+\varepsilon(|-\boldsymbol{k}+\boldsymbol{q} / 2|)+\left|w_{q}\right|\right\}^{-1} .
$$

We now assume a free electron model. The Fermi surface is a sphere of the radius (momentum):

$$
k_{F} \equiv\left|\boldsymbol{k}_{F}\right|=\left(2 m_{1} \varepsilon_{F}\right)^{1 / 2},
$$

where $m_{1}$ represents the effective mass of an electron. The energy $\varepsilon(|\boldsymbol{k}|)$ is given by

$$
\varepsilon(|\boldsymbol{k}|) \equiv \varepsilon_{\boldsymbol{k}}=\frac{|\boldsymbol{k}|^{2}-\left|\boldsymbol{k}_{F}\right|^{2}}{2 m_{1}} .
$$

The prime on the $k$-integral in Equation (11) means the restriction:

$$
0<\varepsilon(|\boldsymbol{k}+\boldsymbol{q} / 2|), \quad \varepsilon(|-\boldsymbol{k}+\boldsymbol{q} / 2|)<\hbar \omega_{D} .
$$

We may choose the polar axis along $\boldsymbol{q}$ as shown in Figure 1. The integration with respect to the azimuthal angle simply yields the factor $2 \pi$. The $k$-integral can then be expressed by

$$
\frac{(2 \pi \hbar)^{3}}{v_{0}}=4 \pi \int_{0}^{\frac{\pi}{2}} \mathrm{~d} \theta \sin \theta \int_{k_{F}+\frac{1}{2} q \cos \theta}^{k_{F}+k_{D}-\frac{1}{2} q \cos \theta} \frac{k^{2} \mathrm{~d} k}{\left|w_{q}\right|+2 \varepsilon_{k}+\left(4 m_{1}\right)^{-1} q^{2}}
$$

where $k_{D}$ is given by

$$
k_{D} \equiv m_{1} \hbar \omega_{D} k_{F}^{-1}
$$

After performing the integration and taking the small- $q$ and small- $\left(k_{D} / k_{F}\right)$ limits, we obtain

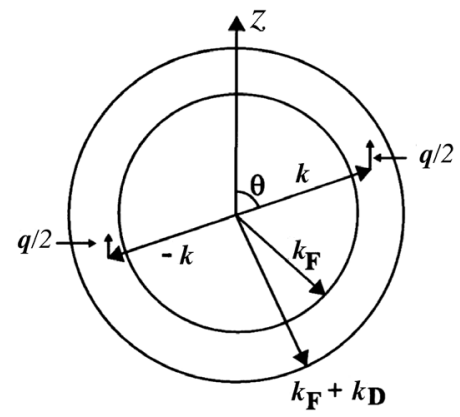

Figure 1. The range of the interaction variables $(k, \theta)$ is restricted to the circular shell of thickness $k_{D}$. 


$$
w_{q}=w_{0}+\frac{v_{F}}{2} q,
$$

where the pairon ground-state energy $w_{0}$ is given by

$$
w_{0}=\frac{-2 \hbar \omega_{D}}{\exp \left\{2 / v_{0} D(0)\right\}-1}, \quad v_{0} \equiv V_{0} / \Omega .
$$

As expected, the zero-momentum pairon has the lowest energy. The excitation energy is continuous with no energy gap. Equation (17) was first obtained by Cooper (unpublished) and it is recorded in Schrieffer's book (Ref. [6], Equations (2)-(15)). The energy $w_{q}$ increases linearly with momentum $q(=|\boldsymbol{q}|)$ for small $q$. This behavior arises since the pairon density of states is strongly reduced with increasing momentum $q$ and dominates the $q^{2}$-increase of the kinetic energy. This linear dispersion relation means that a pairon moves like a massless particle with a common speed $v_{F} / 2$.

The center-of-mass (CM) of pairons move as bosons. We can show this as follows. Second-quantized operators for a pair of "electrons" (i.e., "electron" pairons) are defined by

$$
B_{12}^{\dagger} \equiv B_{\boldsymbol{k}_{1} \uparrow \boldsymbol{k}_{2} \downarrow}^{\dagger} \equiv C_{1}^{\dagger} c_{2}^{\dagger}, \quad B_{34}=c_{4} c_{3}
$$

where $c^{\prime}$ 's and $c^{\dagger}$ 's satisfy the Fermi anti-commutation rules:

$$
\left[c_{k}, c_{k^{\prime}}^{\dagger}\right]_{+} \equiv c_{k} c_{k^{\prime}}^{\dagger}+c_{k^{\prime}}^{\dagger} c_{k}=\delta_{k, k^{\prime}}, \quad\left[c_{k}, c_{k^{\prime}}\right]_{+}=0 .
$$

Using Equation (20) we compute commutation relations for pair operators and obtain

$$
\begin{gathered}
{\left[B_{12}, B_{34}\right] \equiv B_{12} B_{34}-B_{34} B_{12}=0} \\
{\left[B_{12}, B_{34}^{\dagger}\right]= \begin{cases}1-n_{1}-n_{2} & \text { if } \boldsymbol{k}_{1}=\boldsymbol{k}_{3} \text { and } \boldsymbol{k}_{2}=\boldsymbol{k}_{4} \\
c_{2} C_{4}^{\dagger} & \text { if } \boldsymbol{k}_{1}=\boldsymbol{k}_{3} \text { and } \boldsymbol{k}_{2} \neq \boldsymbol{k}_{4} \\
c_{1} C_{3}^{\dagger} & \text { if } \boldsymbol{k}_{1} \neq \boldsymbol{k}_{3} \text { and } \boldsymbol{k}_{2}=\boldsymbol{k}_{4} \\
0 & \text { otherwise }\end{cases} } \\
\left(B_{12}\right)^{2} \equiv B_{12} B_{12}=0,
\end{gathered}
$$

where

$$
n_{1} \equiv c_{\boldsymbol{k}_{1} \uparrow}^{\dagger} c_{\boldsymbol{k}_{1} \uparrow}, \quad n_{2} \equiv c_{\boldsymbol{k}_{2} \downarrow}^{\dagger} c_{\boldsymbol{k}_{2} \downarrow}
$$

are number operators for electrons. Using Equations (19)-(24), we obtain

$$
n_{12}^{2} \equiv\left(B_{12}^{\dagger} B_{12}\right)^{2}=B_{12}^{\dagger}\left(1-n_{1}-n_{2}+B_{12}^{\dagger} B_{12}\right) B_{12}=B_{12}^{\dagger} B_{12} \equiv n_{12} .
$$

Hence $\left(n_{12}^{2}-n_{12}\right)\left|n_{12}^{\prime}\right\rangle=n_{12}^{\prime}\left(n_{12}^{\prime}-1\right)\left|n_{12}^{\prime}\right\rangle=0$, indicating that the eigenvalues $n_{12}^{\prime}$ of $n_{12}$ are $n_{12}^{\prime}=0$ or 1 . Thus, the number operator in the $\boldsymbol{k}-\boldsymbol{q}$ representation $n_{\boldsymbol{k} \boldsymbol{q}} \equiv B_{\boldsymbol{k} \boldsymbol{q}}^{\dagger} B_{\boldsymbol{k} \boldsymbol{q}}$ with both $\boldsymbol{k}$ and $\boldsymbol{q}$ specified, has the eigenvalues 0 or 1 .

We now introduce

$$
B_{q} \equiv \sum_{k} B_{k q}
$$

and calculate $\left[B_{q}, n_{q}\right]$ and obtain

$$
\left[B_{q}, n_{q}\right]=\sum_{k}\left(1-n_{k+q / 2}-n_{-k+q}\right) B_{k q}=B_{q}, \quad\left[n_{q}, B_{q}^{\dagger}\right]=B_{q}^{\dagger},
$$

from which it follows straightforwardly that the eigenvalues $n_{q}^{\prime}$ of $n_{q}$ are: [7]

$$
n_{q}^{\prime}=0,1,2, \cdots
$$

with the eigenstates

$$
|0\rangle, \quad|1\rangle=B_{q}^{\dagger}|0\rangle, \quad|2\rangle=B_{q}^{\dagger} B_{q}^{\dagger}|0\rangle, \cdots .
$$




\section{Ginzburg-Landau Equation at $0 \mathrm{~K}$}

Let us take a three dimensional (3D) superconductor such as tin ( $\mathrm{Sn}$ ) and lead (Pb). Both metals form face-centered cubic (fcc) crystals. They are in superconducting states at $0 \mathrm{~K}$.

The system ground-state wave function $\Psi_{0}(\boldsymbol{r})$ is a constant in the normalization volume $\Omega$ and vanishes at the boundary:

$$
\Psi_{0}(\boldsymbol{r})=\left\{\begin{array}{l}
\text { constant within the volume } \Omega, \\
0 \text { at the boundary. }
\end{array}\right.
$$

We may assume a periodic rectangular-box with side-lengths $\left(L_{x}, L_{y}, L_{z}\right)$ along the cubic lattice,

$$
L_{j}=N_{j} a, \quad N_{j} \gg 1, \quad j=x, y, z,
$$

where $a$ is the lattice constant.

We introduce a one-body density operator $n$ and the density matrix $n_{a b}$ for the treatment of a many-particle system. The density operator $n$ can be expanded in the form:

$$
n=\sum_{\mu}|\mu\rangle P_{\mu}\langle\mu|, \quad P_{\mu} \geq 0
$$

where $\left\{P_{\mu}\right\}$ denote the relative probabilities that particle states $\{\mu\}$ are occupied. It is customary to adopt the following normalization condition:

$$
n=\sum_{\mu} P_{\mu}=\operatorname{tr}\{n\}=N
$$

where the symbol "tr" means a one-body trace and $N$ is the particle number. The density operator $n$ is Hermitean:

$$
n^{\dagger} \equiv n
$$

Let us introduce kind of a square root density operator $u$ such that

$$
n=u u^{\dagger} \text {. }
$$

This $u$ is not Hermitean but $u u^{\dagger}=n$ is. We will show that the revised G-L wave function $\Psi_{\sigma}(\boldsymbol{r})$ is connected with

$$
\Psi_{\sigma}(\boldsymbol{r})=\langle\boldsymbol{r}|u| \sigma\rangle
$$

where $\sigma$ denotes the condensed pairon state. For a running ring super current [8] we may choose

$$
\sigma=p_{m}=\frac{2 \pi}{L} m, \quad m=0, \pm 1, \pm 2, \cdots,
$$

where $L$ is the ring circumference. The $|m|$ are very small numbers $(|m| \ll N)$. The energies of the excited states $m$ are practically the same as the ground state $(m=0)$ since $|m| \ll N$ and $L \gg a$. The excited states are semi-stable because $N$ particles must be redistributed when going from an excited state $m$ to the ground state 0 . The natural decay times are measured in days.

Let us introduce boson field operators $\psi(\boldsymbol{r}, t)$ and $\psi^{\dagger}(\boldsymbol{r}, t)$ which satisfy the Bose commutation rules:

$$
\begin{aligned}
& {\left[\psi(\boldsymbol{r}), \psi^{\dagger}\left(\boldsymbol{r}^{\prime}\right)\right] \equiv \psi(\boldsymbol{r}) \psi^{\dagger}\left(\boldsymbol{r}^{\prime}\right)-\psi^{\dagger}\left(\boldsymbol{r}^{\prime}\right) \psi(\boldsymbol{r})=\delta^{(3)}\left(\boldsymbol{r}-\boldsymbol{r}^{\prime}\right),} \\
& {\left[\psi(\boldsymbol{r}), \psi\left(\boldsymbol{r}^{\prime}\right)\right]=\left[\psi^{\dagger}(\boldsymbol{r}), \psi^{\dagger}\left(\boldsymbol{r}^{\prime}\right)\right]=0, \quad \text { (the time } t \text { omitted). }}
\end{aligned}
$$

where $\delta^{(3)}\left(\boldsymbol{r}-\boldsymbol{r}^{\prime}\right) \equiv \delta\left(x-x^{\prime}\right) \delta\left(y-y^{\prime}\right) \delta\left(z-z^{\prime}\right)$ and $\delta\left(x-x^{\prime}\right)$ is Dirac's delta-function.

We take a system characterized by many-boson Hamiltonian $H$ :

$$
H=\int \mathrm{d}^{3} r \psi^{\dagger}(\boldsymbol{r}) h(\boldsymbol{r},-i \hbar \partial / \partial \boldsymbol{r}) \psi(\boldsymbol{r})+\frac{1}{2} \int \mathrm{d}^{3} r \int \mathrm{d}^{3} r^{\prime} \psi^{\dagger}(\boldsymbol{r}) \psi^{\dagger}\left(\boldsymbol{r}^{\prime}\right) \phi\left(\left|\boldsymbol{r}-\boldsymbol{r}^{\prime}\right|\right) \psi\left(\boldsymbol{r}^{\prime}\right) \psi(\boldsymbol{r})
$$

where $h(\boldsymbol{r}, \boldsymbol{p})$ is a single-boson Hamiltonian and $\phi\left(\left|\boldsymbol{r}-\boldsymbol{r}^{\prime}\right|\right)$ is a pair potential energy. The field equation obtained from the Heisenberg equation of motion is 


$$
i \hbar \frac{\partial \psi(\boldsymbol{r}, t)}{\partial t}=[\psi(\boldsymbol{r}, t), H]=h(\boldsymbol{r},-i \hbar \partial / \partial \boldsymbol{r}) \psi(\boldsymbol{r}, t)+\int \mathrm{d}^{3} r^{\prime} \phi\left(\left|\boldsymbol{r}-\boldsymbol{r}^{\prime}\right|\right) \psi^{\dagger}\left(\boldsymbol{r}^{\prime}, t\right) \psi\left(\boldsymbol{r}^{\prime}, t\right) \psi(\boldsymbol{r}, t) .
$$

We note that the field equation is nonlinear in the presence of a pair potential.

We can exprress $\langle\boldsymbol{r}|$ and $|\boldsymbol{r}\rangle$ by

$$
\left\langle\boldsymbol{r}\left|=\left\langle\Phi_{0}|\psi(\boldsymbol{r}), \quad| \boldsymbol{r}\right\rangle=\psi^{\dagger}(\boldsymbol{r})\right| \Phi_{0}\right\rangle,
$$

where $\psi(\boldsymbol{r})$ and $\psi^{\dagger}(\boldsymbol{r})$ are boson operators and $\left\langle\Phi_{0}\right|$ and $\left|\Phi_{0}\right\rangle$ represent vacuum state vectors satisfying

$$
\left\langle\Phi_{0}\left|\psi^{\dagger}(\boldsymbol{r})=\psi(\boldsymbol{r})\right| \Phi_{0}\right\rangle=0, \quad\left\langle\Phi_{0} \mid \Phi_{0}\right\rangle=1 .
$$

In the Heisenberg picture (HP) the boson states are time-independent and boson operators $\psi$ and $\psi^{\dagger}$ move following the field Equation (40).

The single particle Hamiltonian $h$ contains the kinetic energy $h_{0}(\boldsymbol{p})$, which depends on the momentum $\boldsymbol{p}$ only, and the boson-condensation Hamiltonian $h_{e}$ which arises from the phonon exchange attraction. The ground state wave function $\Psi_{0}(\boldsymbol{r})$ is flat as seen from Equation (30). There are no gradients and no material currents. Hence, we obtain

$$
h_{0}(\boldsymbol{p}) \Psi_{\sigma}^{(0)}(\boldsymbol{r})=h_{0}(-i \hbar \partial / \partial \boldsymbol{r}) \Psi_{0}^{(0)}(\boldsymbol{r})=0,
$$

where the superscript (0) means the ground state average.

The ground-state energy of the pairon is negative and is given by $w_{0}$ in Equation (18). Hence we may choose

$$
\alpha_{0} \Psi_{\sigma}^{(0)}(\boldsymbol{r})=w_{0} \Psi^{(0)}(\boldsymbol{r}), \quad \alpha_{0}=w_{0}<0 .
$$

The pairon has charge magnitude $2 e$ and a size. For $\mathrm{Pb}$ the pairon linear size is about $10^{3} \AA$. Because of the Colomb repulsion and Pauli's exclusion principle two pairons repel each other at short distances. We may represent this repulsion by a point-like pair potential:

$$
\phi\left(\left|\boldsymbol{r}-\boldsymbol{r}^{\prime}\right|\right)=v_{0} \delta^{(3)}\left(\left|\boldsymbol{r}-\boldsymbol{r}^{\prime}\right|\right), \quad v_{0}(\text { constant })>0 .
$$

Using this and the random phase (factorization) approximation we obtain

$$
\begin{aligned}
& v_{0} \int \mathrm{d}^{3} r^{\prime} \delta^{(3)}\left(\boldsymbol{r}-\boldsymbol{r}^{\prime}\right) \Psi_{\sigma}^{\dagger}\left(\boldsymbol{r}^{\prime}\right) \Psi_{\sigma}\left(\boldsymbol{r}^{\prime}\right) \Psi_{\sigma}(\boldsymbol{r})=v_{0}\left|\Psi_{\sigma}(\boldsymbol{r})\right|^{2} \Psi_{\sigma}(\boldsymbol{r}) . \\
& \text { (t-dependence and superscript omitted) }
\end{aligned}
$$

Gathering the results (43), (44) and (46), we obtain

$$
i \hbar \frac{\partial \Psi_{\sigma}(\boldsymbol{r}, t)}{\partial t}=w_{0} \Psi(\boldsymbol{r}, t)+v_{0}\left|\Psi_{0}(\boldsymbol{r}, t)\right|^{2} \Psi_{\sigma}(\boldsymbol{r}, t) .
$$

For the steady state the time derivative vanishes, yielding

$$
w_{0} \Psi_{\sigma}(\boldsymbol{r})+v_{0}\left|\Psi_{\sigma}(\boldsymbol{r})\right|^{2} \Psi_{\sigma}(\boldsymbol{r})=0 .
$$

This is precicely, the GL equation, Equation (3) with $\alpha_{0}=w_{0}$ and $\beta_{0}=v_{0}$.

In our derivation we assumed that pairons move as bosons, which is essential. Bosonic pairons can multiply occupy the condensed momentum state while fermionic superelectrons cannot. The correct density condition (6) instead of (5) must therefore be used.

\section{Discussion}

We derived the GL equation from first principles. In the derivation we found that the particles that are described by the GL wave function $\Psi(\boldsymbol{r})$ must be bosons. We take the view that $\Psi_{\sigma}(\boldsymbol{r})$ represents the bosonically condensed pairons. This explains the quantum nature of the GL wave function.

The nonlinearity of the GL equation arises from the point-like repulsive inter-pairon interaction. In 1950 when Ginzburg and Landau published their work, the Cooper pair (pairon) was not known. They simply assumed the 
superelectron model.

Our microscopic derivation allows us to interpret the expansion parameters as follows. The $\alpha_{0}$ represents the pairon condensation energy, and $\beta_{0}$ the repulsive interaction strength:

$$
\beta_{0}=\text { constant }>0 \text {. }
$$

BCS showed [4] that the ground-state energy $W$ for the BCS system is

$$
W=\hbar \omega_{D} D(0) w_{0}, \quad w_{0} \equiv \frac{-2 \hbar \omega_{D}}{\exp \left\{2 / v_{0} D(0)\right\}-1},
$$

where $D(0)$ is the density of states per spin at the Fermi energy and $w_{0}$ the pairon ground-state energy. Hence we obtain Equation (44): $\alpha_{0}=w_{0}<0$ at $T=0$.

In the original work [1] GL considered a superconductor in the vicinity of the critical temperature $T_{c}$, where $\left|\Psi_{\sigma}\right|^{2}$ is small. Gorkov [9]-[11] used Green's functions and interrelated the GL and the BCS theory near $T_{c}$. We derived the original GL equation by examining the superconductor at $0 \mathrm{~K}$ from the condensed pairons point of view. The transport property of a superconductor below $T_{c}$ is dominated by the Bose-condensed pairons. Since there is no distribution, the qualitative property of the condensed pairons cannot change with temperature. The pairon size (the minimum of the coherence length derivable directly from the GL equation) naturally exists. There is only one supercondensate whose behavior is similar for all temperatures below $T_{c}$; only the density of condensed pairons can change. Thus, there is a quantum nonlinear equation (48) for $\Psi_{\sigma}(\boldsymbol{r})$ valid for all temperateures below $T_{c}$. The pairon energy spectrum below $T_{c}$ has a discrete ground-state energy, which is separated from the energy continuum of moving pairons [5]. Inspection of the pairon energy spectrum with a gap suggests that

$$
\alpha_{0}=-\varepsilon_{g}(T)<0, \quad T_{c}>T .
$$

Solving Equation (48) with Equation (6), we obtain

$$
n_{0}(T)=\left|\Psi_{\sigma}\right|^{2}=\beta_{0}^{-1} \varepsilon_{g}(T),
$$

indicating that the condensed density $n_{0}(T)$ is proportional to the pairon energy gap $\varepsilon_{g}(T)$.

We now consider an ellipsoidal macroscopic sample of a type I superconductor below $T_{c}$ subject to a weak magnetic field $\boldsymbol{H}$ applied along its major axis. Because of the Meissner effect, the magnetic fluxes are expelled from the body, and the magnetic energy is higher by $\frac{1}{2} \mu_{0} H^{2} \Omega$ in the super state than in the normal state. If the field is sufficiently raised, the sample reverts to the normal state at a critical field $H_{c}$, which can be computed in terms of the free-energy expression (1) with the magnetic field included. We obtain after using Equations (6) and (50)

$$
H_{c} \simeq\left(\mu_{0} \beta_{0}\right)^{-1 / 2} \varepsilon_{g}(T) \propto n_{0}(T),
$$

indicating that the measurements of $H_{c}$ give the T-dependent $n_{0}(T)$ approximately. The field-induced transition corresponds to the evaporation of condensed paions, and not to their break-up into electrons. Moving pairons by construction have negative energies while quasi electrons have positive energies. Thus, the moving pairons are more numerous at the lowest temperatures, and they are dominant elementary excitations. Since the contribution of the moving pairons was neglected in the above calculation, Equation (51) contains approximation, see below.

We stress that the pairon energy gap $\varepsilon_{g}$ is distinct from the BCS energy gap $\Delta$, which is the solution of

$$
1=v_{0} D(0) \int_{0}^{\hbar \omega_{D}} \mathrm{~d} \varepsilon \frac{1}{\left(\varepsilon^{2}+\Delta^{2}\right)^{1 / 2}} \tanh \left[\frac{\left(\varepsilon^{2}+\Delta^{2}\right)^{1 / 2}}{2 k_{B} T}\right] .
$$

In the presence of a supercondensate the energy-momentum relation for an unpaired (quasi) electron changes:

$$
\varepsilon_{k} \equiv\left|k^{2} /\left(2 m_{0}\right)-\varepsilon_{F}\right| \rightarrow E_{k} \equiv\left(\varepsilon_{k}^{2}+\Delta^{2}\right)^{1 / 2} .
$$

Since the density of condensed pairons changes with the temperature $T$, the gap $\Delta$ is $T$-dependent and is 
determined from Equation (54) (originated in the BCS energy gap equation). Two unpaired electrons can be bound by the phonon-exchange attraction to form a moving pairon whose energy $\tilde{w}_{0}$ is given by

$$
\begin{gathered}
\tilde{w}_{q}=\tilde{w}_{0}+\frac{1}{2} v_{F} q<0, \\
1=v_{0} D(0) \int_{0}^{\hbar \omega_{D}} \mathrm{~d} \varepsilon\left\{\left|\tilde{w}_{0}\right|+2\left(\varepsilon^{2}+\Delta^{2}\right)^{1 / 2}\right\}^{-1} .
\end{gathered}
$$

Note that $\tilde{w}_{0}$ is $T$-dependent since $\Delta$ is. At $T_{c}, \Delta=0$ and the lower band edge $\tilde{w}_{0}$ is equal to the pairon ground-state energy $w_{0}$. We may then write

$$
\tilde{w}_{q}=w_{0}+\varepsilon_{g}(T)+\frac{1}{2} v_{F} q, \quad \varepsilon_{g}(T) \equiv \tilde{w}_{0}-w_{0} \geq 0 .
$$

We call $\varepsilon_{g}(T)$ the pairon energy gap. The two gaps $\left(\Delta, \varepsilon_{g}\right)$ have similar $T$-behavior; they are zero at $T_{c}$ and they both grow monotonically as temperature is lowered. The rhs of Equation (54) is a function of $\left(T, \Delta^{2}\right)$; $T_{c}$ is a regular point such that a small variation $\delta T \equiv T_{c}-T$ generates a small variation in $\Delta^{2}$. Hence we obtain

$$
\Delta(T) \simeq b\left(T_{c}-T\right)^{1 / 2}, \quad T_{c}-T \ll T_{c}, \quad b=\text { constant } .
$$

Using similar arguments we get from Equations (57)-(59)

$$
\varepsilon_{g}(T) \simeq c\left(T_{c}-T\right), \quad T_{c}-T \ll T_{c}, \quad c=\text { constant } .
$$

As noted earlier, moving pairons have finite (zero) energy gaps in the super (normal) states, which makes Equation (53) approximate. But the gaps disappear at $T_{c}$, and hence the linear-in- $\left(T_{c}-T\right)$ behavior should hold for the critical field $H_{c}$ :

$$
H_{c}=d\left(T_{c}-T\right), \quad T_{c}-T \ll T_{c}, \quad d=\text { constant },
$$

which is supported by experimental data. Tunneling and photo absorption data [12]-[16] appear to support the linear law in Equation (60).

In the original GL theory [1], the following signs and T-dependence of the expansion parameters $\left(\alpha_{0}, \beta_{0}\right)$ near $T_{c}$ were assumed and tested:

$$
\alpha_{0} \simeq-c\left(T_{c}-T\right)<0, \quad \beta_{0}=\text { constant }>0,
$$

all of which are reestablished by our microscopic calculations.

\section{Conclusions}

In summary we reached a significant conclusion that the GL equation is valid for all temperatures below $T_{c}$. The most important results in the GL theory include GL's introduction of a coherent length [1] and Abrikosov's prediction of a vortex structure [17], both concepts holding not only near $T_{c}$ but for all temperatures below $T_{c}$.

In the present work the time evolution of the system is described through the field equation for the boson operators $\psi(\boldsymbol{r}, t)$ and $\psi^{\dagger}(\boldsymbol{r}, t)$. The resulting equation

$$
i \hbar \frac{\partial \Psi_{\sigma}(\boldsymbol{r}, t)}{\partial t}=h(\boldsymbol{r},-i \hbar \partial / \partial \boldsymbol{r}) \Psi_{\sigma}(\boldsymbol{r}, t)+v_{0}\left|\Psi_{0}(\boldsymbol{r}, t)\right|^{2} \Psi_{\sigma}(\boldsymbol{r}, t)
$$

may be useful in deriving the Josephson-Feynman equation and describing dynamical Josephson effect [18] [19]. Operators $u$ and $u^{\dagger}$ are non-Hermitean, see Equation (35). Hence, a off-diagonal long range order simply arises from the definition of $\Psi_{0}(\boldsymbol{r}) \equiv\left\langle\boldsymbol{r}_{0}|u| \sigma\right\rangle$.

GL treated the effect of the magnetic field applied based on the super electron model. We shall treat this effect based on the moving pairons model separately.

\section{References}

[1] Ginzburg, V.L. and Landau, L.D. (1950) Journal of Experimental and Theoretical Physics (USSR), 20, 1064.

[2] Landau, L.D. and Lifshitz, E.M. (1980) Statistical Physics, Part I. 3rd Edition, Pergamon Press, Oxford, 171-174. 
[3] Cooper, L.N. (1956) Physical Review, 104, 1189. http://dx.doi.org/10.1103/PhysRev.104.1189

[4] Bardeen, J., Cooper, L.N. and Schrieffer, J.R. (1957) Physical Review, 108, 1175. http://dx.doi.org/10.1103/PhysRev.108.1175

[5] Fujita, S., Ito, K. and Godoy, S. (2009) Quantum Theory of Conducting Matter: Superconductivity. Springer, New York, 77-79, 133-138. http://dx.doi.org/10.1007/978-0-387-88211-6

[6] Schrieffer, J.R. (1964) Theory of Superconductivity. Benjamin, New York.

[7] Dirac, P.A.M. (1958) Principle of Quantum Mechanics. 4th Edition, Oxford University Press, London, 136-138.

[8] File, J. and Mills, R.G. (1963) Physical Review Letters, 10, 93. http://dx.doi.org/10.1103/PhysRevLett.10.93

[9] Gorkov, I. (1958) Soviet Physics-JETP, 7, 505.

[10] Gorkov, I. (1959) Soviet Physics-JETP, 9, 1364.

[11] Gorkov, I. (1960) Soviet Physics—JETP, 10, 998.

[12] Glaever, I. (1960) Physical Review Letters, 5, 147. http://dx.doi.org/10.1103/PhysRevLett.5.147

[13] Glaever, I. (1960) Physical Review Letters, 5, 464. http://dx.doi.org/10.1103/PhysRevLett.5.464

[14] Glaever, I. and Megerle, K. (1961) Physical Review, 122, 1101. http://dx.doi.org/10.1103/PhysRev.122.1101

[15] Glover III, R.E. and Tinkham, M. (1957) Physical Review, 108, 243. http://dx.doi.org/10.1103/PhysRev.108.243

[16] Biondi, M.A. and Garfunkel, M. (1959) Physical Review, 116, 853. http://dx.doi.org/10.1103/PhysRev.116.853

[17] Abrikosov, A.A. (1957) Soviet Physics—JETP, 5, 1174.

[18] Josephson, B.D. (1962) Physics Letters, 1, 251-253. http://dx.doi.org/10.1016/0031-9163(62)91369-0

[19] Josephson, B.D. (1964) Reviews of Modern Physics, 36, 216. http://dx.doi.org/10.1103/RevModPhys.36.216 
Scientific Research Publishing (SCIRP) is one of the largest Open Access journal publishers. It is currently publishing more than 200 open access, online, peer-reviewed journals covering a wide range of academic disciplines. SCIRP serves the worldwide academic communities and contributes to the progress and application of science with its publication.

Other selected journals from SCIRP are listed as below. Submit your manuscript to us via either submit@scirp.org or Online Submission Portal.
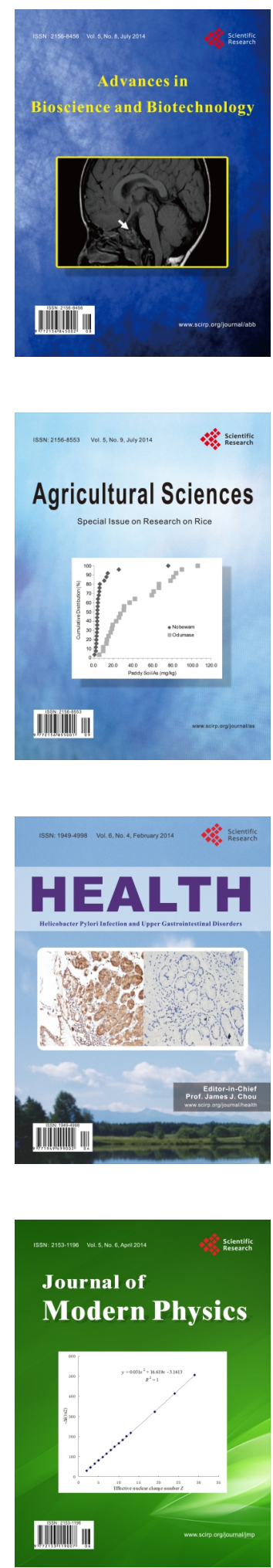
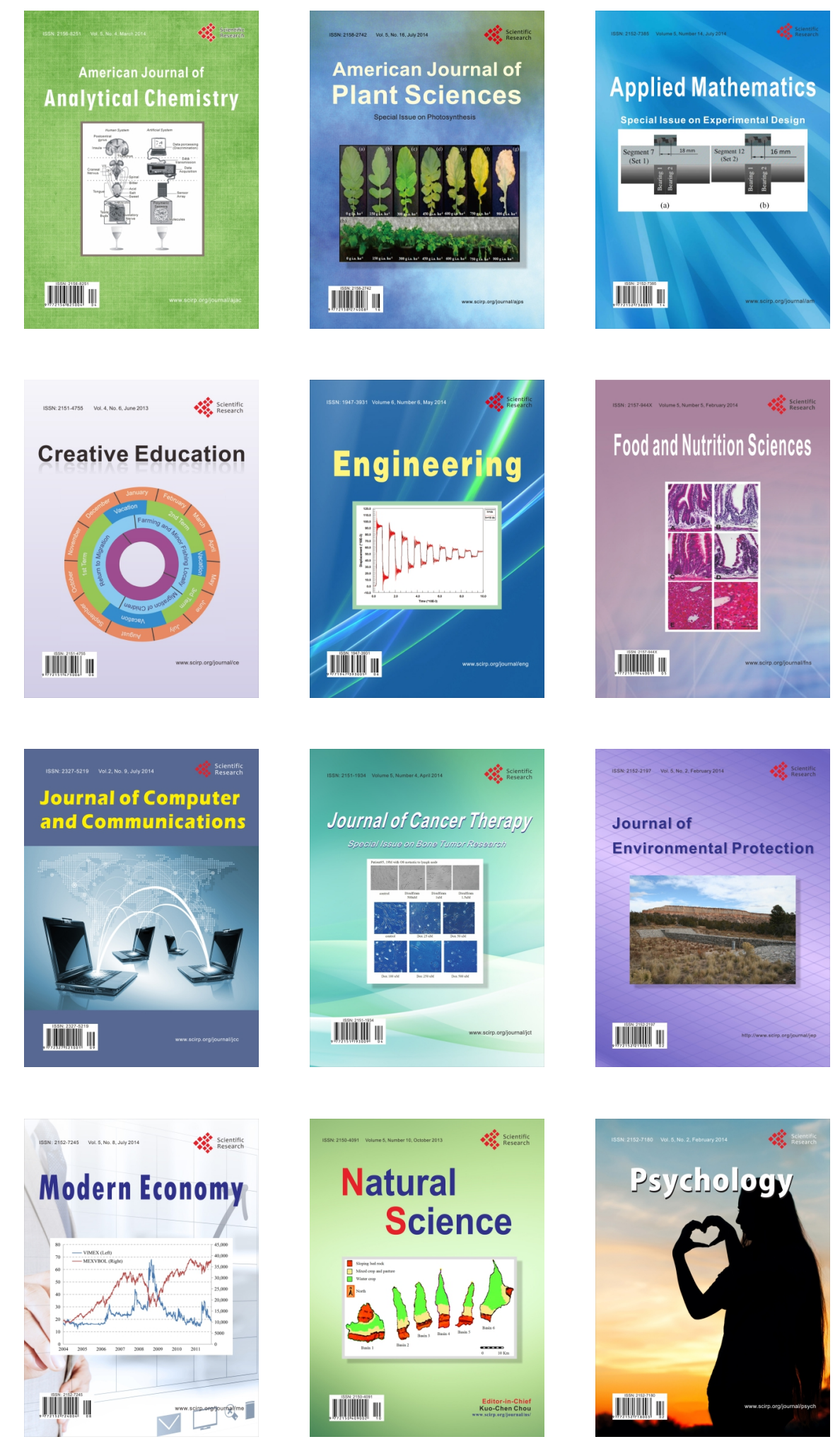